\title{
Diagnosis and management of testosterone deficiency syndrome in men: clinical practice guideline
}

\author{
Alvaro Morales CM MD, Richard A. Bebb MD, Priya Manjoo MD MSc, Peter Assimakopoulos MD, \\ John Axler MD, Christine Collier PhD, Stacy Elliott MD, Larry Goldenberg CM OBC MD, Irv Gottesman MD, \\ Ethan D. Grober MD MEd, Gordon H. Guyatt MD MSc, Daniel T. Holmes MD, Jay C. Lee MD; Canadian Men's \\ Health Foundation Multidisciplinary Guidelines Task Force on Testosterone Deficiency
}

CMAJ Podcasts: author interview at https://soundcloud.com/cmajpodcasts/150033-guide

See also page 1342 and www.cmaj.ca/lookup/doi/10.1503/cmaj.151208

$\mathrm{T}$ estosterone deficiency syndrome, also known as late-onset hypogonadism, is a clinical and biochemical syndrome that can occur in men in association with advancing age. The condition is characterized by deficient testicular production of testosterone. It may affect multiple organ systems and can result in substantial health consequences. ${ }^{1}$

The management of testosterone deficiency syndrome in men is associated with much controversy, creating confusion and reluctance among health practitioners in dealing with the subject. A small number of professional bodies ${ }^{2,3}$ have published guidelines on the topic, yet a multidisciplinary guideline with specific Canadian content did not exist. This is relevant because there are important differences in health care systems compared with other jurisdictions in North America and Europe, including familiarity with the concepts of adult testosterone deficiency, accessibility to some laboratory tests and availability of certain formulations of testosterone. In this article, we identify and address the knowledge gaps across disciplines to assist a variety of health professionals in their clinical decision-making in managing testosterone deficiency syndrome.

In addition to the Canada-wide survey conducted as part of our needs assessment, two recent studies support the need for an increased effort in educating physicians on the basics of managing testosterone deficiency in men. Researchers in Ontario found that 1 in 90 men over age 65 years was being prescribed testosterone replacement therapy, but only $6 \%$ of these patients had a conclusive diagnosis of hypogonadism. ${ }^{4}$ These findings are further validated by a large, long-term study conducted in the United Kingdom and the United States that showed that as many as $40 \%$ of men were obtaining testosterone without a documented biological deficiency. ${ }^{5}$
This guideline is intended to address clinical questions surrounding the diagnosis of testosterone deficiency and the appropriate use of testosterone replacement therapy in the management of these patients. The document places a high priority on the identification and treatment of symptomatic men, and the improvement of patient outcomes. (Appendix 1 contains the fulltext guideline, available at www.cmaj.ca/lookup/ suppl/doi:10.1503/cmaj.150033/-/DC1.)

\section{Scope}

Based on the results of a broad survey of practising physicians in Canada conducted as part of our needs assessment (Appendix 1), the Canadian Men's Health Foundation provided support and assembled a multidisciplinary group - the Canadian Men's Health Foundation Multidisciplinary Guidelines Task Force on Testosterone Deficiency — to develop a clinical practice guideline for the management of testosterone deficiency

\section{KEY POINTS}

- Diagnosis of testosterone deficiency syndrome requires the presence of the clinical manifestations of testosterone deficiency, together with documented testosterone levels below the local laboratory ranges.

- Treatment is recommended for testosterone deficiency syndrome; the choice of treatment is based on product safety, efficacy, tolerability, cost and the absence of contraindications.

- Testosterone replacement therapy is appropriate in men with testosterone deficiency syndrome who have cardiovascular disease or are at risk of cardiovascular disease.

- Hypogonadal men with successfully treated prostate cancer may be candidates for testosterone supplementation; these patients require referral to a specialist, because treatment involves close monitoring by a physician with expertise in the risks and benefits of testosterone therapy.

- Regular monitoring for clinical and biochemical response, and for adverse effects, to testosterone replacement therapy is essential, particularly during the first year of treatment. 
syndrome. The foundation recognized that this area is pertinent to several clinical disciplines; therefore, a range of specialists (i.e., clinical biochemists, endocrinologists, epidemiologists, family physicians and urologists) was considered for the task force to ensure the guideline was representative and would reflect a broad perspective.

The wider scope and target audience of clinicians includes Canadian primary care physicians, general internists and internal medicine subspecialists (endocrinologists and geriatricians), and urologists. A further group of interest involves clinical biochemists, psychiatrists, nurse practitioners and pharmacists dealing with men at and beyond middle age with manifestations of testosterone deficiency syndrome.

The population addressed in this article comprises men with clinical manifestations compatible with testosterone deficiency syndrome and laboratory confirmation of testosterone deficiency in Canada. These are usually men with multiple comorbidities for whom issues related to the diagnosis, management and follow-up of testosterone deficiency syndrome require a patient-centred approach.

\section{Methods}

The task force met to identify guideline sections and writing responsibilities, in accordance with their clinical or laboratory knowledge, practice and expertise. Two task force members were assigned primary responsibility for writing each section. The authors of each section proposed clinical questions phrased according to the PICO (Patient, Intervention, Comparator and Outcome) format and following the Grading of Recommendations Assessment, Development and Evaluation (GRADE) approach for evaluation of the evidence and development of the recommendations (www.gradeworkinggroup.org). ${ }^{6,7}$

The task force followed the six domain principles of the Appraisal of Guidelines for Research and Evaluation (AGREE II) recommendations for guideline development, ${ }^{8}$ which ensured that potential biases were addressed and that the recommendations are not only internally

\section{Box 1: Grading of recommendations?}

- The strength of the recommendations (weak or strong) is based on the quality of the supporting evidence, the level of uncertainty between desirable and undesirable clinical effects or diagnostic reliability, and therapeutic preferences.

- Strong recommendations are indicated by the phrase "we recommend," whereas weak recommendations are indicated by the phrase "we suggest."

- The quality of the evidence relied on the appraisal of the likelihood that additional research will modify a recommendation. It was rated as very low, low, moderate or high quality. and externally valid, but also realistic and practical for use by health professionals. Grading of the recommendations is summarized in Box $1 .^{7}$

With the aid of an endocrinologist on the task force (P.M.), a librarian with recognized expertise affiliated with the DeGroote Institute at McMaster University conducted a systematic search of MEDLINE, Embase, the Cochrane Central Register of Controlled Trials (CENTRAL) and PubMed for clinical trials and observational studies using key words related to the topic. The search was conducted for the period January 2009 until April 2014. Reports from meta-analyses, practice guidelines, clinical conferences and major reviews were also examined, and papers were manually searched for additional references. We were permitted to include relevant studies published after the search period, while the guideline document was under editorial assessment and in response to peer reviewers' comments. This included articles up to April 2015. An independent pharmacist with experience in conducting and evaluating literature searches assessed the search strategy used and the results of the literature search.

The task force used an evidence-based approach to acknowledge limitations in the published literature before generating recommendations. The authors of each section assessed the literature pertaining to clinically important outcomes in their respective areas and assigned a grade (high, moderate, low or very low) to describe the quality of the evidence using the procedure outlined by the GRADE Working Group. ${ }^{7,9}$ In brief, randomized controlled trials were initially rated as high-quality and observational studies as low-quality evidence. The grade was then adjusted after consideration of the risk of bias, study limitations, consistency of results, directness of the evidence, precision of the results and publication bias across the body of relevant literature. Where appropriate, evidence summary tables were created for transparency.

The section authors then generated recommendations that were graded as either "strong" or "weak" after assessing the quality of the evidence, the uncertainty between the desirable and undesirable effects of the intervention, variabilities in patient values and preferences, as well as the potential impact on use of resources. All recommendations were phrased in accordance to the GRADE format.

The sections of the guideline were presented to the task force as a whole for review and comments at an initial all-day meeting; they were circulated after the meeting for further comments. The comments were collated and, together with the recommendations and evidence for the rec- 


\section{Box 2. Abridged recommendations for the diagnosis and management of testosterone deficiency syndrome*}

\section{Diagnosis}

1. We recommend a thorough history and physical examination, instead of the exclusive reliance on standard questionnaires, to identify patients requiring biochemical testing (strong recommendation; moderate-quality evidence).

2. The initial biochemical test should be total testosterone level measured in serum samples taken in the morning; determinations of bioavailable testosterone or free testosterone should be restricted to patients with equivocally low total testosterone levels (strong recommendation; high-quality evidence).

3. We recommend that sample collection for testosterone measurement occur between 7 am and 11 am, or within 3 hours after waking in the case of shift workers (strong recommendation; moderate-quality evidence).

4. Testosterone levels should be measured with the use of testosterone assays traceable to internationally recognized standardized reference material; commercial assays should be certified by the testosterone standardization program of the US Centers for Disease Control and Prevention (strong recommendation; high-quality evidence).

5. Measurement of sex hormone-binding globulin with calculated free or bioavailable testosterone should be restricted to men with symptoms of testosterone deficiency and equivocally low testosterone levels (strong recommendation; moderate-quality evidence).

6. We recommend investigation for secondary or reversible causes of hypogonadism in all men with testosterone deficiency syndrome (strong recommendation; moderate-quality evidence).

7. We recommend investigation for testosterone deficiency syndrome and treatment with testosterone in men with anemia or sarcopenia of undetermined origin (strong recommendation; moderate-quality evidence).

8. We suggest investigation and testosterone treatment for men with depression refractory to standard treatment, as well as for men taking glucocorticoid or opioid therapy and men with HIV infection if they have experienced weight loss (weak recommendation; low-quality evidence).

9. In men with erectile dysfunction and no other manifestations of testosterone deficiency syndrome, we suggest investigation only after a trial of phosphodiesterase type 5 (PDE-5) inhibitors has failed (weak recommendation; low-quality evidence).

\section{Treatment}

10. We recommend that men with documented testosterone deficiency syndrome and no contraindications should receive treatment with testosterone (strong recommendation; high-quality evidence).

11. Men with testosterone deficiency syndrome and stable cardiovascular disease are candidates for testosterone treatment (weak recommendation; low-quality evidence).

12. We suggest that men with testosterone deficiency syndrome and localized prostate cancer and no evidence of active disease receive testosterone therapy (weak recommendation; low-quality evidence).

13. We suggest testosterone treatment for men with testosterone deficiency syndrome and mild-to-moderate symptoms due to benign prostatic hypertrophy (weak recommendation; very-low-quality evidence).

14. We recommend against testosterone replacement therapy in men with metastatic prostate cancer (strong recommendation; moderate- to high-quality evidence).

15. Men with a history of breast cancer are not candidates for testosterone replacement therapy (weak recommendation; moderatelevel of evidence).

16. We recommend against testosterone replacement therapy in men more interested in maintaining fertility over symptomatic improvement (strong recommendation; high-quality evidence).

17. We recommend treatment with a PDE-5 inhibitor in men with testosterone deficiency syndrome and persistent erectile dysfunction that is adequately treated with testosterone (strong recommendation; high-quality evidence).

18. In men with a clinical picture strongly suggestive of testosterone deficiency syndrome but testosterone levels in the low-normal range, we suggest a therapeutic trial with testosterone (weak recommendation; very-low-quality evidence).

\section{Monitoring}

19. We recommend assessment of response and adverse effects at three and six months after onset of therapy (strong recommendation; high-quality evidence).

20. Testosterone levels should be assessed at three and six months after onset of therapy and then annually thereafter if stable (weak recommendation; low-quality evidence).

21. We recommend assessment of hematocrit at baseline, at three and six months and then annually (strong recommendation; highquality evidence).

22. We recommend determinations of prostate-specific antigen at baseline, at three and six months and then annually (strong recommendation; moderate- to low-quality evidence).

23. A digital rectal examination should be performed at baseline, at six months and then annually following onset of treatment (weak recommendation; very-low-quality evidence).

24. Testosterone replacement therapy should be discontinued in men with testosterone deficiency syndrome if contraindications to therapy arise or if there is no improvement after an adequate therapeutic trial (weak recommendation; moderate-quality evidence).

*For a more comprehensive list of recommendations, including expanded commentary and literature references, see Appendix 1 (available at www.cmaj.ca/lookup/ suppl/doi:10.1503/cmaj.150033/-/DC1). 
ommendations, were presented at a second allday meeting for additional review and comments. An independent medical writer who participated at the meetings was employed to achieve uniformity in the initial drafts of the documents.

Following final approval by the task force as a whole, the graded recommendations were reviewed by two members with recognized expertise in epidemiology and guideline development (G.H.G. and P.M.) for further refinement and consistency.

\section{Recommendations}

The task force recognizes that the management of testosterone deficiency syndrome remains highly controversial and that, in many instances, the evidence continues to be of doubtful quality. However, health professionals must deal with patients presenting with clinical manifestations and biochemical confirmation of the diagnosis.

An abridged version of the recommendations and their grading is shown in Box 2. The literature review process, detailed recommendations, evidence profiles and summary-of-findings tables are presented in Appendix 1.

\section{Diagnosis}

The manifestations of testosterone deficiency are not specific, vary in onset and severity, and need not all be present to reach a diagnosis. ${ }^{10}$ Sexual symptoms and fatigue are the earliest and most common presentations. ${ }^{11}$ Other symptoms (e.g., depression, sleep alterations, poor concentration) and metabolic disorders (e.g., type 2 diabetes mellitus, obesity) are seen at borderline testosterone levels. The symptoms are frequently subtle and may be affected by factors such as age, general health, comorbidities and medications, as well as systemic illness and environmental factors. ${ }^{12}$ Patient history is therefore an important component in the diagnostic process. The signs and symptoms associated with testosterone deficiency syndrome are presented in Box 3, and associated features on history are listed in Table 6 in Appendix 1.

\section{Box 3: Signs and symptoms associated with testosterone deficiency} syndrome ${ }^{1}$

- Sexual: Decreased libido; erectile dysfunction; decreased frequency of morning erections; decreased performance

- Somatic: Increased visceral body fat/obesity; decreased lean muscle mass; decreased strength; fatigue/loss of energy; decreased physical activity/ vitality; low bone mineral density; anemia; flushes; loss of facial, axillary and pubic hair/slow beard growth; decline in general feeling of well-being

- Psychological: Depression/depressed mood; mood changes; irritability; inability to concentrate; insomnia/sleep disturbances
In the diagnosis of testosterone deficiency, the physical examination bears similar shortcomings to those of patient history, mainly because of lack of specificity. Gonadal atrophy may be present, as well as a decrease in pubic and facial hair. A decrease in muscle mass and increase in visceral fat may also be present. The sequela of osteoporosis becomes evident only when the hypogonadism has been present for long periods. ${ }^{12}$ None of these findings, alone or in combination, are sufficient to make a diagnosis of testosterone deficiency syndrome. However, the history and results of the examination collectively would make a strong case for the presumptive diagnosis of the syndrome, thus identifying patients who require biochemical testing.

To facilitate the diagnosis of testosterone deficiency, a number of screening questionnaires have been developed to identify men with low testosterone levels. In general, however, these tools have high sensitivity but low specificity (see Appendix 1 for details). Despite their economy and ease of administration, their availability on the Internet and the opportunity they provide patients to self-diagnose, the questionnaires cannot be relied upon in the absence of confirmatory history, physical findings and laboratory test results.

The task force recommends that the initial biochemical test be total testosterone level measured in a blood sample taken in the morning (or within three hours of waking in the case of shift workers), and that determinations of bioavailable testosterone or free testosterone be restricted to patients found to have equivocal low total testosterone levels (Box 2).

The clinical diagnosis of testosterone deficiency syndrome can be challenging because clinicians frequently face situations in which the laboratory results remain inconclusive in the presence of symptoms. Thus, it has been suggested that, in the presence of a convincing clinical picture but uncertain laboratory results, a therapeutic trial of testosterone supplementation (of perhaps three months) is an acceptable diagnostic approach. ${ }^{13,14}$

An algorithm outlining an approach to the diagnosis of testosterone deficiency is available in Figure 1.

\section{Treatment}

Once testosterone deficiency is diagnosed, treatment involves testosterone supplementation in most cases. Notable exceptions include secondary hypogonadism amenable to therapy aimed at improving pituitary function, metabolic alterations that may respond to other measures (see the section on Alternative and Complimentary 
Treatment in Appendix 1) and potential contraindications. An algorithm providing a step-bystep therapeutic plan is shown in Figure 2.

The goal of testosterone treatment is improvement in symptoms and achievement of eugonadal levels of testosterone, about in the mid-normal range for healthy young men $(14-17.5 \mathrm{nmol} / \mathrm{L}){ }^{15}$ Because there are large variations between individuals, symptom improvement should be the primary objective, and higher or lower serum testosterone concentrations may be acceptable in patients with a positive response to treatment. ${ }^{16}$

Monitoring is an integral and mandatory part of the management of hypogonadism. The potential benefits and harms that can be expected from testosterone supplementation are listed in Table 1. ${ }^{17,18}$ Lack of or an inadequate clinical response to testosterone treatment should call for changes in the form of delivery, the dose or the frequency of administration. A persistent failure to respond to testosterone supplementation requires a reassessment of the diagnosis or consideration for referral to a more specialized facility.

In Canada, the available products for testosterone therapy include short-acting injectable testosterone (testosterone enanthate, testosterone cypionate and testosterone propionate), oral testosterone undecanoate, transdermal testosterone patches, transdermal testosterone gel 1\% (hydroalcoholic gel, and hydroalcoholic gel with pentadecalactone) and axillary transdermal testosterone solution $2 \%$. Intramuscular injection of testosterone propionate is used infrequently. Compounded testosterone products are available at many compounding pharmacies in Canada, but there are no published data on the safety and efficacy of these products.

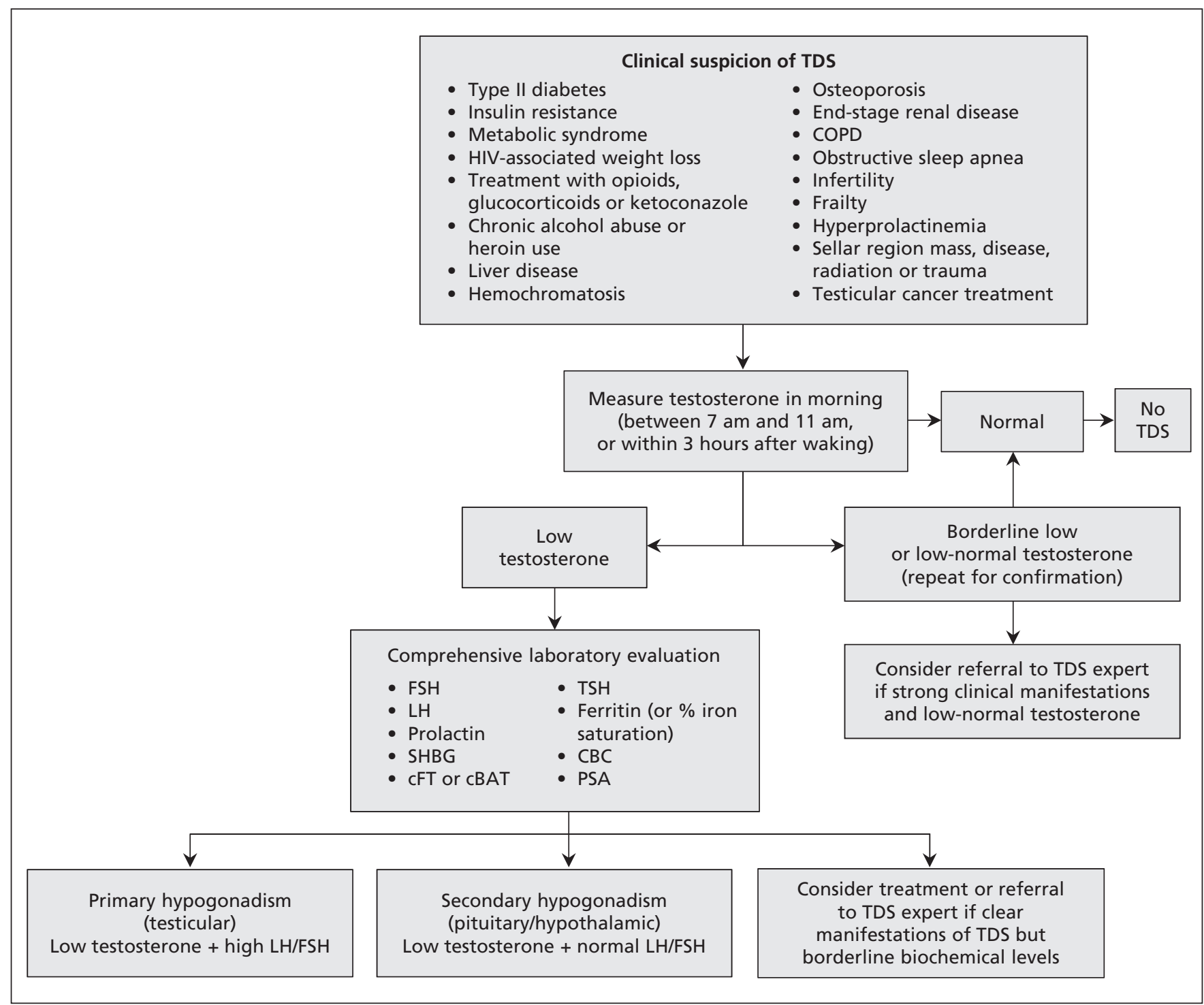

Figure 1: Algorithm for the diagnosis of testosterone deficiency syndrome (TDS). CBAT = calculated bioavailable testosterone, CBC = complete blood count, $\mathrm{CFT}=$ calculated free testosterone, COPD = chronic obstructive pulmonary disease, FSH = follicle-stimulating hormone LH = luteinizing hormone, PSA = prostate-specific antigen, SHBG = sex hormone-binding globulin, TSH = thyroid-stimulating hormone. 
The choice of product for testosterone replacement therapy should be a topic of discussion between the physician, the patient, and the patient's caregiver, if appropriate. Factors affecting this choice include safety, efficacy, tolerability, availability, preference and cost. More information on advantages and disadvantages of available products, including costs, is outlined in Tables 7 and 8 of Appendix 1.

Because of the particularly controversial nature of the subject and the fluidity of the literature, two recommendations on testosterone treatment in men with stable cardiovascular disease and prostate cancer have been selected for expanded description of the evidence supporting them.

Appendix 1 contains more information on the role of testosterone therapy in testosterone deficiency associated with alterations in body composition and metabolic issues, as well as in other chronic illnesses, such as HIV infection and depression.

\section{Men with stable cardiovascular disease}

Short-term studies of testosterone treatment have shown symptomatic improvement in hypogonadal men with coronary artery disease or congestive heart failure. ${ }^{19,20}$ However, evidence regarding a beneficial or harmful effect of testosterone replacement therapy on hard outcomes such as myocardial infarction, stroke and sudden cardiac death is lacking. The results of several large meta-analyses have generally not sup- ported a causal role between testosterone treatment and cardiovascular events. ${ }^{21-24}$ These metaanalyses are limited by the quality of the included studies, the heterogeneity of the testosterone levels in the populations assessed, and the use of different doses and routes of administration of testosterone replacement products.

Therefore, the task force made a weak recommendation, based on low-quality evidence, that testosterone replacement therapy in men with cardiovascular disease be restricted to those with stable disease, only after a discussion of the potential risks and benefits (Box 2). The recommendations took into account the positions of both Health Canada and the US Food and Drug Administration. For more information, see Appendix 1.

\section{Men with prostate cancer}

The conventional belief that testosterone replacement therapy is contraindicated in the presence of advanced prostate cancer is supported by robust evidence, ${ }^{25}$ thus meriting a strong recommendation against its use (Box 2). Conversely, concerns about testosterone supplementation promoting the development and growth of prostate cancer and benign hyperplasia have long been based on extrapolations more so than on real proof or verification. Recently, these perceptions have been challenged by several small studies supporting the "saturation model," indicating that the prostate is sen-

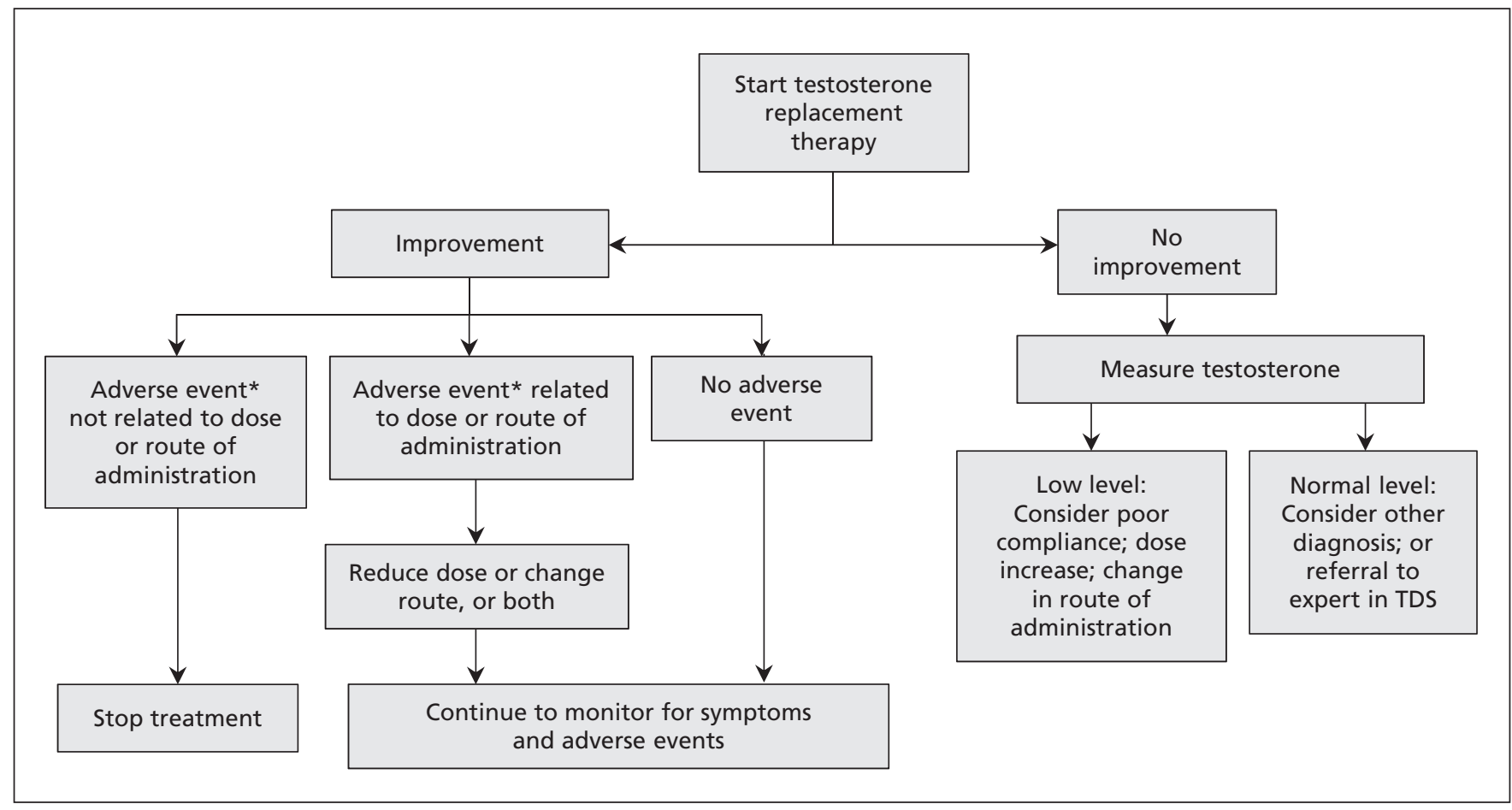

Figure 2: Algorithm for the treatment of testosterone deficiency syndrome (TDS). *Report adverse events to MedEffect Canada (www. hc-sc.gc.ca/dhp-mps/medeff/report-declaration/index-eng.php\#a2). 
sitive to androgens to a certain point beyond which further growth does not occur despite increasing levels of testosterone. ${ }^{26}$ Further studies are necessary, and verification may change in the future; therefore, these recommendations only merited a weak ranking with very-low- to low-quality evidence.

However, hypogonadal men with successfully treated prostate cancer may be candidates for testosterone supplementation. These patients require referral to a specialist, because treatment involves close monitoring by a physician with expertise in the risks and benefits of testosterone therapy. For more information, see Appendix 1.

\section{Implementation}

The Canadian Men's Health Foundation is developing a communications plan for the guideline among its public campaigns to increase awareness of testosterone deficiency issues related to men's health. Knowledge translation tools that include the diagnosis and treatment algorithms and the list of abridged recommendations included in the guideline will be distributed among the audience of health professionals identified earlier. The algorithms and the abridged recommendations constitute a readily available resource for most professionals whose clinical practice includes a sizable number of men over 50 years of age, among whom testosterone deficiency syndrome is most prevalent.

\section{Resource implications}

The prevalent confusion about the diagnosis of testosterone deficiency, the inappropriate use of testosterone replacement therapy and the recent reports of potentially increased risk of serious adverse effects with the use of testosterone replacement therapy indicate an important need for guidance among health professionals in the management of testosterone deficiency. The task force did not consider in its mandate the costs associated with the implementation of the guideline, in part because of the different provincial policies related to coverage of laboratory testing and specific drug formulations (Table 8 in Appendix 1 provides information on the costs of the various preparations).

\section{Other guidelines}

This guideline is largely in agreement with the Endocrine Society's clinical practice guideline on testosterone therapy in men with androgen deficiency syndromes published in $2010,{ }^{2}$ which was a thorough, evidence-based assessment using the GRADE system to define the quality of the evidence and the strength of the recommendations. Two years earlier, a set of recommendations with less strict standards for assessment of the evidence was published simultaneously in various journals by an international expert group of endocrinologists and urologists. ${ }^{3}$ A Canadian publication authored by physicians of various in-

Table 1: Potential benefits and harms of testosterone supplementation in men with testosterone deficiency syndrome ${ }^{* 17,18}$

\begin{tabular}{|c|c|c|}
\hline Organ system & Benefits & Harms \\
\hline Erectile function/libido & Improvement & None \\
\hline Depression/mood/fatigue & Improvement & Aggressive behaviour \\
\hline Erythropoiesis & Increase in hematocrit & $\begin{array}{l}\text { Increased risk of polycythemia, } \\
\text { embolism }\end{array}$ \\
\hline Skeletal muscle & Increase in fat-free mass & None \\
\hline Bone metabolism & Prevention of osteoporosis & None \\
\hline Cardiovascular system & $\begin{array}{l}\text { Improvement in congestive heart } \\
\text { failure, exercise capacity }\end{array}$ & $\begin{array}{l}\text { Increased risk of thromboembolic } \\
\text { cardiovascular events }\end{array}$ \\
\hline \multicolumn{3}{|l|}{ Prostate } \\
\hline $\begin{array}{l}\text { Benign prostatic } \\
\text { hyperplasia }\end{array}$ & $\begin{array}{l}\text { None beyond manifestations of } \\
\text { testosterone deficiency syndrome }\end{array}$ & $\begin{array}{l}\text { Marginal increase in volume and } \\
\text { prostate-specific antigen level }\end{array}$ \\
\hline $\begin{array}{l}\text { Cancer (metastatic or } \\
\text { high risk of recurrence) }\end{array}$ & Absolute contraindication & Recurrence and rapid progression \\
\hline $\begin{array}{l}\text { Cancer (localized and } \\
\text { treated) }\end{array}$ & $\begin{array}{l}\text { None beyond manifestations of } \\
\text { testosterone deficiency syndrome }\end{array}$ & $\begin{array}{l}\text { Potential exacerbation of } \\
\text { subclinical residual cancer }\end{array}$ \\
\hline Testicle & $\begin{array}{l}\text { None beyond manifestations of } \\
\text { testosterone deficiency syndrome }\end{array}$ & $\begin{array}{l}\text { Atrophy or impairment of } \\
\text { spermatogenesis }\end{array}$ \\
\hline
\end{tabular}


terests and backgrounds, but aimed primarily at urologists, appeared in $2010 .{ }^{27}$ Neither of these latter two publications used a recognized system for evaluation of the evidence.

In 2012, the European Association of Urology published a guideline on male hypogonadism that was developed by European genitourinary surgeons. ${ }^{28}$ The levels of evidence were graded in accordance with a rating scheme adapted from the Oxford Centre for EvidenceBased Medicine's levels of evidence. The 2012 guideline is fundamentally in agreement with the recommendations in our guideline, but it does not include more recent publications. Furthermore, it was published at a time when highly controversial views on testosterone and cardiovascular health had not yet received such heightened attention and before the new concepts on testosterone and prostate carcinogenesis were widely publicized. Also, it was intended for a specific audience of European genitourinary surgeons and is not easily accessible to most Canadian health professionals.

\section{Gaps in knowledge}

Our needs-assessment survey indicated that about one-quarter of physicians were not comfortable with the diagnosis and management of testosterone deficiency (Appendix 1). It was of considerable interest that $61 \%$ of the respondents (including a portion of those who considered themselves comfortable with the diagnosis and management of testosterone deficiency) agreed to be part of a follow-up study. This study will allow for an objective comparative assessment of the impact of the guideline on the target audience.

The management of testosterone deficiency syndrome has been controversial for decades and contributes substantially to the uncertainty among clinicians. Prominent among controversial issues are concerns about the diagnostic accuracy of laboratory tests, and cardiovascular and prostate health. We have addressed these issues in the guideline, based on the latest supporting evidence; however, active research promises to settle part of the controversy in the next few years. With the increased release of new data and the elucidation of answers to remaining clinical questions, it is anticipated that this guideline will be revised within the next three to five years.

\section{Conclusion}

The management of testosterone deficiency syndrome is contentious. The current evidence conclusively shows the importance of an adequate diagnostic approach followed by testosterone supplementation when the diagnosis is reached. The task force did not find compelling evidence for withholding testosterone therapy in symptomatic men who also have cardiovascular disease or localized prostate cancer. In addition, it found a role for testosterone therapy in testosterone deficiency associated with alterations in body composition and metabolic issues, as well as in other chronic illnesses (e.g., HIV infection and depression). The evidence also points to the importance of regular follow-up, frequently within the first year after the start of treatment and less stringent thereafter but uninterrupted for the duration of treatment.

\section{References}

1. Buvat J, Maggi M, Guay A, et al. Testosterone deficiency in men: systematic review and standard operating procedures for diagnosis and treatment. J Sex Med 2013;10:245-84.

2. Bhasin S, Cunningham GR, Hayes FJ, et al.; Task Force, Endocrine Society. Testosterone therapy in adult men with androgen deficiency syndromes: an Endocrine Society clinical practice guideline. J Clin Endocrinol Metab 2010;95:2536-59.

3. Wang C, Nieschlag E, Swerdloff R, et al. Investigation, treatment and monitoring of late-onset hypogonadism in males: ISA, ISSAM, EAU, EAA and ASA recommendations. Eur J Endocrinol 2008; 159:507-14.

4. Piszczek J, Mamdani M, Antoniou T, et al. The impact of drug reimbursement policy on rates of testosterone replacement therapy among older men. PLOS ONE 2014;9:e98003.

5. Layton JB, Li D, Meier CR, et al. Testosterone lab testing and initiation in the United Kingdom and the United States, 2000 to 2011. J Clin Endocrinol Metab 2014;99:835-42.

6. Guidelines for managing reviews and overlapping scope. London (UK): Cochrane Collaboration. Available: http://community. cochrane.org/editorial-and-publishing-policy-resource/guidelines -managing-reviews-overlapping-scope (accessed 2015 Oct. 5).

7. Guyatt GH, Oxman AD, Kunz R, et al. GRADE: going from evidence to recommendations. BMJ 2008;336:1049-51.

8. Brouwers MC, Kho ME, Browman GP, et al.; AGREE Next Steps Consortium. AGREE II: advancing guideline development, reporting and evaluation in health care. CMAJ 2010;182:E839-42.

9. Guyatt GH, Oxman AD, Vist G, et al. GRADE: an emerging consensus on rating quality of evidence and strength of recommendations. BMJ 2008;336:924-6.

10. Cunningham GR, Stephens-Shields AJ, Rosen RC, et al. Association of sex hormones with sexual function, vitality, and physical function of symptomatic older men with low testosterone levels at baseline in the testosterone trials. J Clin Endocrinol Metab 2015;100:1146-55.

11. Hall SA, Esche GR, Araujo AB, et al. Correlates of low testosterone and symptomatic androgen deficiency in a populationbased sample. J Clin Endocrinol Metab 2008;93:3870-7.

12. Wu FC, Tajar A, Beynon JM, et al. Identification of late-onset hypogonadism in middle-aged and elderly men. $N$ Engl $\mathrm{J} \mathrm{Med}$ 2010;363:123-35.

13. Black AM, Day AG, Morales A. The reliability of clinical and biochemical assessment in symptomatic late-onset hypogonadism: Can a case be made for a 3 -month therapeutic trial? BJU Int 2004;94:1066-70.

14. Morley JE. The diagnosis of late life hypogonadism. Aging Male 2007; 10:217-20.

15. Corona G, Rastrelli G, Maggi M. Diagnosis and treatment of lateonset hypogonadism: systematic review and meta-analysis of TRT outcomes. Best Pract Res Clin Endocrinol Metab 2013;27:557-79.

16. Ullah MI, Riche DM, Koch CA. Transdermal testosterone replacement therapy in men. Drug Des Devel Ther 2014;8:101-112.

17. Ruige JB, Ouwens DM, Kaufman JM. Beneficial and adverse effects of testosterone on the cardiovascular system in men. J Clin Endocrinol Metab 2013;98:4300-10.

18. Cunningham GR, Toma SM. Clinical review: Why is androgen replacement in males controversial? J Clin Endocrinol Metab 2011:96:38-52.

19. Stout M, Tew GA, Doll H, et al. Testosterone therapy during exercise rehabilitation in male patients with chronic heart failure who have low testosterone status: a double-blind randomized controlled feasibility study. Am Heart J 2012;164:893-901.

20. Toma M, McAlister FA, Coglianese EE, et al. Testosterone sup- 
plementation in heart failure: a meta-analysis. Circ Heart Fail 2012;5:315-21.

21. Fernández-Balsells MM, Murad MH, Lane M, et al. Clinical review 1: Adverse effects of testosterone therapy in adult men: a systematic review and meta-analysis. J Clin Endocrinol Metab 2010;95:2560-75.

22. Calof OM, Singh AB, Lee ML, et al. Adverse events associated with testosterone replacement in middle-aged and older men: a meta-analysis of randomized, placebo-controlled trials. J Gerontol A Biol Sci Med Sci 2005;60:1451-7.

23. Haddad RM, Kennedy CC, Caples SM, et al. Testosterone and cardiovascular risk in men: a systematic review and meta-analysis of randomized placebo-controlled trials. Mayo Clin Proc 2007; 82:29-39.

24. Corona G, Maseroli E, Rastrelli G, et al. Cardiovascular risk associated with testosterone-boosting medications: a systematic review and meta-analysis. Expert Opin Drug Saf 2014;13:1327-51.

25. Huggins C, Hodges CV. Studies on prostatic cancer. I. The effect of castration, of estrogen and of androgen injection on serum phosphatases in metastatic carcinoma of the prostate. 1941. J Urol 2002;167:948-51, discussion 952.

26. Roddam AW, Allen NE, Appleby P, et al. Endogenous sex hormones and prostate cancer: a collaborative analysis of 18 prospective studies. J Natl Cancer Inst 2008;100:170-83.

27. Morales A, Bella AJ, Chun S, et al. A practical guide to diagnosis, management and treatment of testosterone deficiency for Canadian physicians. Can Urol Assoc J 2010;4:269-75.

28. Dohle GR, Arver S, Bettocchi C, et al. Guidelines on male hypogonadism. Arnhem (The Netherlands): European Association of Urology; 2012. Available: http://uroweb.org/wp-content/uploads/ EAU-Guidelines-Male-Hypogonadism-2015.pdf (accessed 2014 Sept. 20).

Competing interests: Alvaro Morales received speaker fees from AbbVie for a presentation at the Hamilton Academy in January 2013; he received consultant fees from AbbVie for review of information on a new drug (not related to the current topic) in the summer of 2012. Richard Bebb has received speaker fees from Abbott and Eli Lilly. Peter Assimakopoulos has received lecture fees from Abbott and is on the advisory board for Eli Lilly. Stacy Elliott has received speaker fees from Eli Lilly and Abbott and is on their advisory boards. Ethan Grober received a research grant from Eli Lilly and has received speaker and consulting fees from Abbott, Paladin and Actavis. Daniel Holmes has received speaker fees from Immunodiagnostic Systems for presentations on mineralocorticoid hypertension at the 2013 Endocrine Society meeting. Jay Lee has received speaker fees from Abbott and Eli Lilly. No competing interests were declared by the other authors.

Affiliations: Department of Urology (Morales), Queen's University, Kingston, Ont.; Division of Endocrinology and
Department of Urologic Sciences (Bebb), University of British Columbia and St. Paul's Hospital, Vancouver, BC; Division of Endocrinology (Manjoo), University of British Columbia, and Department of Medicine (Manjoo), Vancouver Island Health Authority, Vancouver, BC; McGill University and Jewish General Hospital (Assimakopoulos), Montréal, Que.; Department of Family and Community Medicine (Axler), University of Toronto, Toronto, Ont.; Department of Pathology and Molecular Medicine (Collier), Queen's University, Kingston, Ont.; Departments of Psychiatry (Elliott) and Urologic Sciences (Elliott, Goldenberg), University of British Columbia, Vancouver, BC; Canadian Men's Health Foundation (Goldenberg), Vancouver, BC; Faculty of Medicine (Gottesman), University of Toronto, Credit Valley Hospital, Toronto, Ont.; Division of Urology (Grober), Department of Surgery, University of Toronto, Mount Sinai Hospital and Women's College Hospital, Toronto, Ont.; Departments of Clinical Epidemiology and Biostatistics and of Medicine (Guyatt), McMaster University, Hamilton, Ont.; Department of Pathology and Laboratory Medicine (Holmes), University of British Columbia and St. Paul's Hospital, Vancouver, BC; Division of Urology (Lee), Department of Surgery, University of Calgary, Calgary, Alta.

Contributors: All of the task force members contributed substantially to the content and development of the guideline and presented their sections of the guideline at two separate task force meetings. Gordon Guyatt critically revised the methodology and provided editorial guidance with respect to the recommendations but was not an attendee at the two task force meetings. Final edits to the manuscript were carried out by Alvaro Morales and Priya Manjoo. All of the authors approved the final version of the manuscript to be published and agreed to act as guarantors of the work.

Endorsement: The guideline has been endorsed by the Canadian Urological Association and the Canadian Society of Endocrinology and Metabolism.

Funding: Funding for the guideline was provided by the Canadian Men's Health Foundation. The funding body's views did not influence the content of the guideline. The views expressed in this guideline are those of the authors and do not necessarily represent those of the Canadian Men's Health Foundation.

Acknowledgements: The authors thank Core Health Services Inc. for providing organizational and editorial assistance in the development of this guideline, Bryan Simpson PharmD for medical writing assistance and Rachel Couban MA MISt for literature searches. 